\title{
HEART RATE VARIABILITY ANALYSIS TOOL FOR EVALUATION OF AUTONOMIC NERVOUS SYSTEM FUNCTION
}

\author{
Michal GÁLA*, Jana KADLECOVÁ*, Zuzana VIŠŇOVCOVÁ** \\ * Department of Electromagnetic and Biomedical Engineering, Faculty of Electrical Engineering, \\ University of Žilina, Univerzitná 1, 01026 Žilina, Slovak Republic, tel: +421 41513 2148, mail: michal.gala@ fel.uniza.sk \\ ** Department of Physiology, Jessenius Faculty of Medicine, \\ Comenius University in Bratislava, Mala Hora 4, 03601 Martin, Slovak Republic, tel.: +421 432633 404, \\ mail: visnovcova@gmail.com
}

\begin{abstract}
The software described in this study allows the analysis of heart rate variability, a relatively new method for evaluating autonomic nervous system functioning. Analysis of heart rate variability is based on time-domain methods and methods of nonlinear dynamics. The software provides an array of tools for quick and simple analysis of the autonomic nervous system parameters. We aimed to study complexity and time asymmetry of short-term heart rate variability as an index of complex neurocardiac control in response to stress using symbolic dynamics and time irreversibility methods. ECG was recorded at rest, during and after two stressors (Stroop, arithmetic test) in 70 healthy students. Symbolic dynamics parameters $(0 \mathrm{~V} \%, 1 \mathrm{~V} \%, 2 \mathrm{LV} \%, 2 \mathrm{UV} \%)$, and time irreversibility indices $(P \%, G \%, E)$ were evaluated. Correlation analysis revealed no significant associations between symbolic dynamics and time irreversibility. Concluding, symbolic dynamics and time irreversibility could provide independent information related to alterations of neurocardiac control integrity in stress-related disease.
\end{abstract}

Keywords: heart rate variability, HRV analysis, software, time-domain analysis, nonlinear dynamics

\section{INTRODUCTION}

The autonomic nervous system (ANS) represents the principal rapidly reacting system that controls heart rate in response to stress. The acute mental stress is a well-known stimulus characterized by a shifted balance of the ANS towards sympathetic predominance associated with vagal withdrawal. Recent studies referred to an importance of the study regarding cardiovascular responses to mental load: the exaggerated, but also lower cardiovascular reactivity and delayed recovery time could predict future cardiovascular complications [1][2]. The spontaneous short-term oscillations of heart rate - heart rate variability (HRV) - reflect the cardiac autonomic regulation, in particular parasympathetic inputs. However, the heart rate is controlled by a sophisticated control system resulting in the complex oscillations of the heart rate indicating a healthy and adaptive organism. Thus, the reduction of heart rate control network complexity indicates that the heart rate cannot sufficiently adapt to different requirements; thus, the heart is at higher risk of cardiac events [3][4][5]. The assessment of heart rate period variability allows the indirect and non-invasive investigation of the short-term cardiovascular regulatory mechanisms. The complexity of the short-term cardiovascular control system is due to complicated shortterm heart period dynamics [6]. The analysis of heart rate variability (HRV) is important for several reasons: evaluation of autonomic nervous system regulation, risk stratification for sudden cardiac death, diabetic neuropathy, pharmaceutical evaluations and psychological disorders. HRV is a result of ANS (autonomic nervous system) regulation of the sinoatrial (SA) node. ANS is divided into parasympathetic and sympathetic branches which influence the heart rate (HR) and HRV [7][8]. The aim of this study is to present an easy to use HRV analysis software with a wide variety of time-domain and nonlinear analysis options. The said software offers an effective way to detect nonlinear complexities of living dynamical systems, which is one of the most important problems in biomedicine, physics, etc [10].

\section{METHODS OF HEART RATE VARIABILITY ANALYSIS}

The central control element of the autonomic regulation is the sinus node. Its rhythm is usually derived from onsets of P-waves, but due to the difficulty of Pwaves extraction, the intervals between R-peaks are chosen instead of PP intervals. [9]

This section describes the HRV methods included in our software.

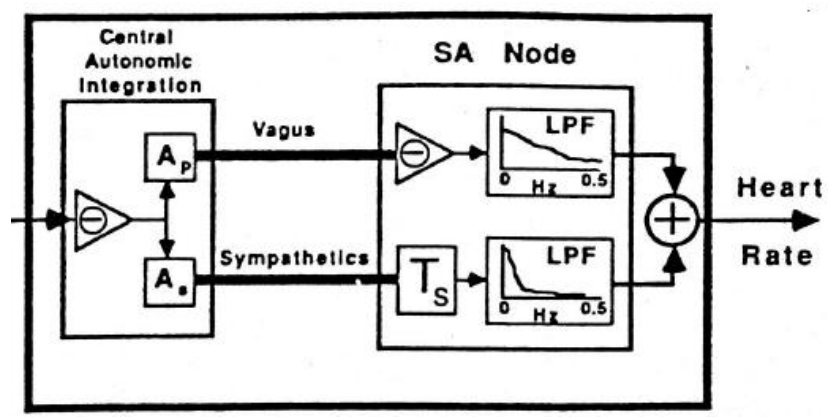

Fig. 1 Heart rate variability controlled by the autonomic nervous system [8]

\subsection{Time-domain methods}

Time-domain measurements are the simplest parameters to be calculated and they can be applied directly to the series of successive RR interval values. In our software we calculated the following time-domain parameters: SDNN, RMSSD, pNN50, HR and mean HR. 
The standard deviation of RR intervals (SDNN index) can be calculated for both short-term and long-term variability while the root mean square of successive differences (RMSSD index) can be used for short-term recordings. Parameter NN50 is the number of successive interbeat intervals that differ more than $50 \mathrm{~ms}$. Index pNN50 is the proportion of NN50 divided by total number of RR intervals. [7]

\subsection{Methods of non-linear dynamics}

Partial processes present in the regulation of ANS can be described reliably by linear methods. However, in view of sinus node activity modulation, HRV cannot be fully described using linear methods. Time and frequency domain parameters are not sufficient in case of investigation of nonlinear complexity of living dynamics [7][12]. Nonlinear methods implemented in our software are: approximate entropy (ApEn), indeces of symbolic analysis (0V\%, 1V\%, 2LV\%, 2UV\%), methods based on time reversibility (Porta index, Guzik index, Ehlers index).

\subsubsection{Aproximate entropy (ApEn)}

The complexity of HRV can be measured by methods based on entropy. One of the methods is approximate entropy. The computation of ApEn index depends on the embedding dimension $L$ and the tolerance $r$.

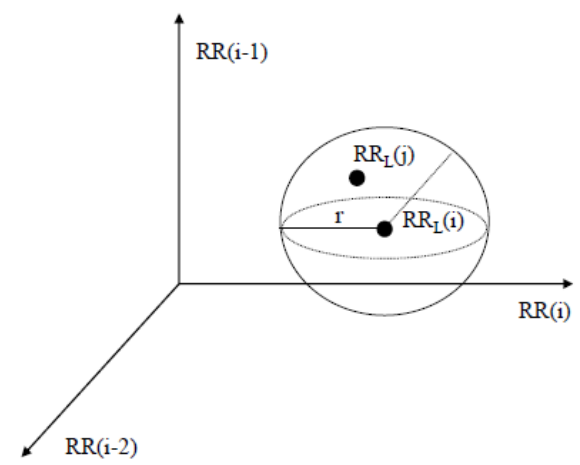

Fig. 2 According to the Euclidean norm, RRL(j) is similar to RRL(i) if RRL(j) lies in a hyper-sphere of radius $r$ centered in RRL(i) [12]

The tolerance $r$ is fixed in relation to parameter SDNN. ApEn is expressed as:

$\Phi(L, r)=-\frac{1}{N-L+1} \sum_{i=1}^{N-L+1} \log \left(C_{i}(L, \mathrm{r})\right)$

where $C_{i}(L, r)$ is the number of points that can be found at distance smaller than $r$ from $\mathrm{x}_{L}(\mathrm{i})$ divided by $N-L+1, N$ number of elements in time series [6].

$\operatorname{ApEn}(L, r)=\Phi(L, r)-\Phi(L-1, r)$

\subsubsection{Methods of symbolic analysis}

Symbolic analysis is an emerging approach in signal processing. This approach is based on the conversion of time series to symbol sequence. The symbols are grouped into "words" and the dynamics of "words" are studied instead of original samples [13]. The analysis method is based on:

- the transformation of short HRV series into a sequence of symbols,

- the construction of "words",

- grouping the "words" into a small number of families,

- the evaluation of the rate of "word families".

The "words" families are:

- $\quad 0 \mathrm{~V}$ - no variation in "word", all symbols are equal,

- 1V- one variation in "word", one symbol is different and two symbols are equal,

- $\quad 2 \mathrm{LV}$ - all symbols are different, symbols form an ascending or descending ramp,

- $2 U V-$ all symbols are different, symbols form a valley or peak,

The indices of symbolic analysis $0 \mathrm{~V} \%, 1 \mathrm{~V} \%, 2 \mathrm{LV} \%$ and $2 \mathrm{UV} \%$ evaluate the rates of occurrence of the families.

\subsubsection{Methods based on time ireversibility}

Time irreversibility analysis investigates the invariance of the statistical properties of a time series after time reversal [14]. This analysis can detect a specific class of nonlinear dynamics - temporal assymetry.

There are several indices proposed to measure the assymetry of a $\Delta R R$ series. We used three traditional irreversibility indices in our analysis software. The considered indices are Porta index, Guzik index and Ehlers index.

\section{A. Porta index}

Porta evaluates the percentage of negative $\Delta R R$ with respect to the total number of $\Delta R R$, which are not equal to zero [14].

$P \%=\frac{N\left(\Delta R R^{-}\right)}{N(\Delta R R \neq 0)} \times 100$

\section{B. Guzik index}

Guzik index evaluates the percentage of the cumulative square values of positive $\Delta R R$ to the cumulative square values of all $\Delta \mathrm{RR}[14]$.

$G \%=\frac{\sum_{i=1}^{N\left(\Delta \mathrm{RR}^{+}\right)} \Delta R R^{+}(\mathrm{i})^{2}}{\sum_{i=1}^{N(\Delta \mathrm{RR})} \Delta \mathrm{RR}(\mathrm{i})^{2}}$

\section{Ehlers index}

Ehlers index evaluates the skewness of the distribution of $\Delta \mathrm{RR}[14]$.

$E=\frac{\sum_{i=1}^{N(\Delta \mathrm{RR})} \Delta \mathrm{RR}(\mathrm{i})^{3}}{\left\{\sum_{i=1}^{N(\Delta \mathrm{RR})} \Delta \mathrm{RR}(\mathrm{i})^{2}\right\}^{3 / 2}}$ 
Table 1 Summary of HRV parameters calculated by our developed software.

\begin{tabular}{|c|c|c|}
\hline Parameter & Units & Description \\
\hline SDNN & [ms] & $\begin{array}{l}\text { The standard deviation of } \mathrm{RR} \\
\text { intervals }\end{array}$ \\
\hline RMSSD & [ms] & $\begin{array}{l}\text { Square root of the mean squared } \\
\text { differences between successive } \\
\text { RR intervals }\end{array}$ \\
\hline pNN50 & {$[\%]$} & $\begin{array}{l}\text { NN50 divided by the total } \\
\text { number of RR intervals }\end{array}$ \\
\hline HR & {$[1 / \mathrm{mi}$} & The mean heart rate \\
\hline ApEn & $\mathrm{n}]$ & Approximate entropy \\
\hline $0 \mathrm{~V}$ & - & The rate of occurrence of $0 \mathrm{~V}$ \\
\hline $1 \mathrm{~V}$ & {$[\%]$} & The rate of occurrence of $1 \mathrm{~V}$ \\
\hline $2 \mathrm{LV}$ & [\%] & The rate of occurrence of $2 \mathrm{LV}$ \\
\hline 2UV & [\%] & The rate of occurrence of $2 \mathrm{UV}$ \\
\hline $\mathrm{P}$ & {$[\%]$} & Porta index \\
\hline G & [\%] & Guzik index \\
\hline $\mathrm{E}$ & {$[\%]$} & Ehlers index \\
\hline
\end{tabular}

\section{ANALYSIS SOFTWARE}

The analysis software has been developed using MATLAB $^{\circledR}$ (The MathWorks, Inc.). It contains all necessary analysis options and also allows the creation of a graphical user interface (GUI).

Our developed application provides a sophisticated array of tools to quickly and accurately analyze heart rate variability.

\subsection{Input data format}

The software supports ASCII text files (*.txt) and input must be beat-to-beat data. In our software we used data measured by microcomputer system DiANS PF8 (Fig. 3). The software allows analysis of the data directly or to save the data to database for later analysis. We used MySQL open source database which is running on our server.

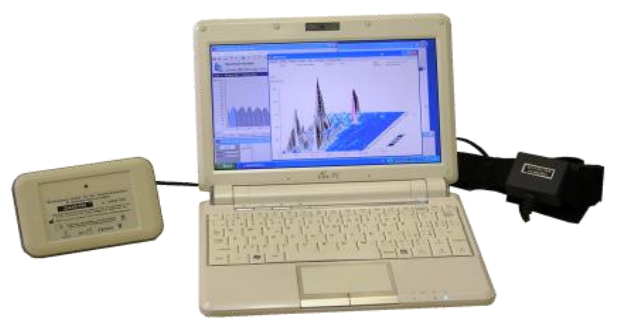

Fig. 3 Microcomputer system for non-invasive examination of the autonomic nervous system DiANS PF8

\subsection{Graphical user interface}

The graphical user interface (GUI) allows:

- to insert RR interval series into MySQL database,

- to load a data series from database and visualize them in a plot,
- to delete a RR interval series from the database,

- to choose the required 300 beats from series for analysis,

- to calculate the indices of HRV analysis,

- to imagine a chosen part of the interval series (300 beats),

- to display the names of recordings in a listbox.

\subsection{Insertion of data series to a database}

After left clicking the button "Insert data series", a dialog box (Fig. 4) is opened where the user can choose data series to be inserted into the database.

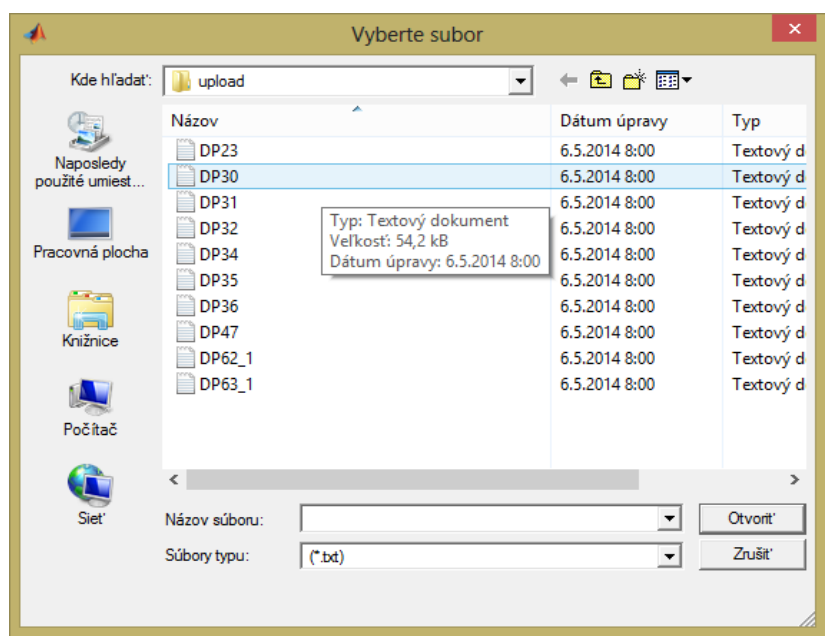

Fig. 4 File selection dialog after clicking "Insert data series" (Slovak language)

Data series saved in the database are accessible for all who have allocated access. Users have to dispose of access to internet connection.

\subsection{Selection and visualisation of data series}

After selecting the filename in listbox (name of RR series), the selected RR series are displayed on the upper RR interval axis. Lower RR interval axis shows first 300 samples of the recording (Fig. 5). The software allows to select any 300 samples for analysis by moving a slider or typing a sample start time in the edit box under the axis. The starting point of selected samples can be set arbitrarily. The selection is visible in the upper axis via two red vertical lines that mark RR interval samples selected for the analysis. Lower axis and vertical lines are automatically updated by moving a slider or changing the sample start time.

Name of the selected data series and number of samples are shown in the top part of GUI (Fig. 5).

It is possible to delete data from the database using the "delete" button. A warning message box is show if the selected data series does not exist in database.

\subsection{Analysis of heart rate variability}

Results of the time-domain and nonlinear analysis are visible after pressing the button "Calculate the indices" in 
the right segment of the application GUI. Index values are displayed in text boxes next to names of indices (Fig. 5).

The GUI is user friendly. The user doesn't need to know patterns for all indices that are calculated in our software (SDNN, RMSSD, pNN50, HR, ApEn, 0V, 1V, 2LV, 2UV, P, G, E). Our analysis tool consists of several modules, one module for one index (e.g. Symbolic analysis module (Table 2)).

Table 2 Symbolic analysis module.

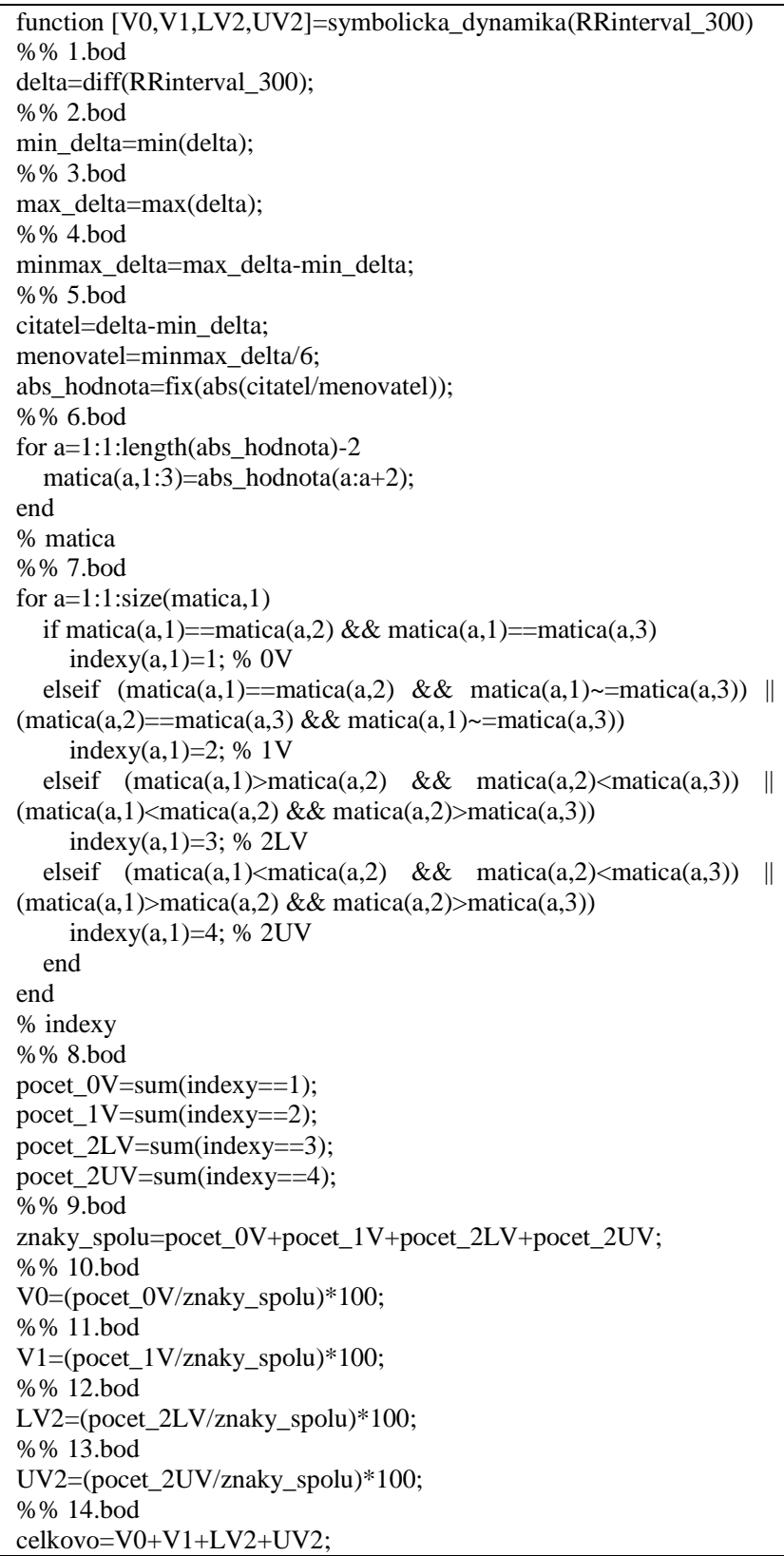

\subsection{Testing}

The described tool was tested on a group of students. The studied group consisted of 70 healthy young students attending the 5th year of Jessenius Faculty of Medicine (39 women, average age: $23.08 \pm 0.17$ yr.). Following exclusion criteria were used during enrolling the subjects: a history of respiratory, endocrinological, cardiovascular, infectious, mental or other diseases potentially influencing HRV (including obesity, underweight, overweight, alcohol or drug abuse). The smokers were excluded from this study. All subjects were instructed not to use substances which affect the cardiovascular system (caffeine, alcohol) for at least 12 hours before the recording. Importantly, because the hormonal changes during menstrual cycle can affect the cardiac autonomic regulation, the females were included in proliferative phase. This study was approved by the Ethics Committee of Jessenius Faculty of Medicine, in accordance with declaration of Helsinki. All subjects were carefully instructed about the study protocol and they gave their informed consent to prior to examination [15]. More information about the study is written in our previous work with name "Complexity and time asymmetry of heart rate variability are altered in acute mental stress" [15].

\section{CONCLUSIONS}

The developed analysis software allows analysis of heart rate variability and measurement of the autonomic nervous system using non-invasive methods. It includes a variety of time-domain and nonlinear HRV parameters.

Our study revealed that qualitative features in complex dynamics of heart rate modulation are altered during mental acute stress. Furthermore, symbolic dynamics indices $0 \mathrm{~V} \%$ and $2 \mathrm{LV} \%$ could reflect a shift in sympathovagal balance in response to stress. Therefore, HRV nonlinear analysis based on symbolic dynamics could provide additional important and mutually independent information related to sophisticated complex neurocardiac integrity in acute/chronic stress. It could help to elucidate the pathway linking health and stress-related disease.

The software is fully operated through an easy to use and intuitive graphical user interface and supports RR interval data formats used for analysis of HRV.

\section{ACKNOWLEDGMENTS}

This paper is supported by the following project: University Science Park of the University of Zilina (ITMS: 26220220184) supported by the Research\&Development Operational Program funded by the European Regional Development Fund. 


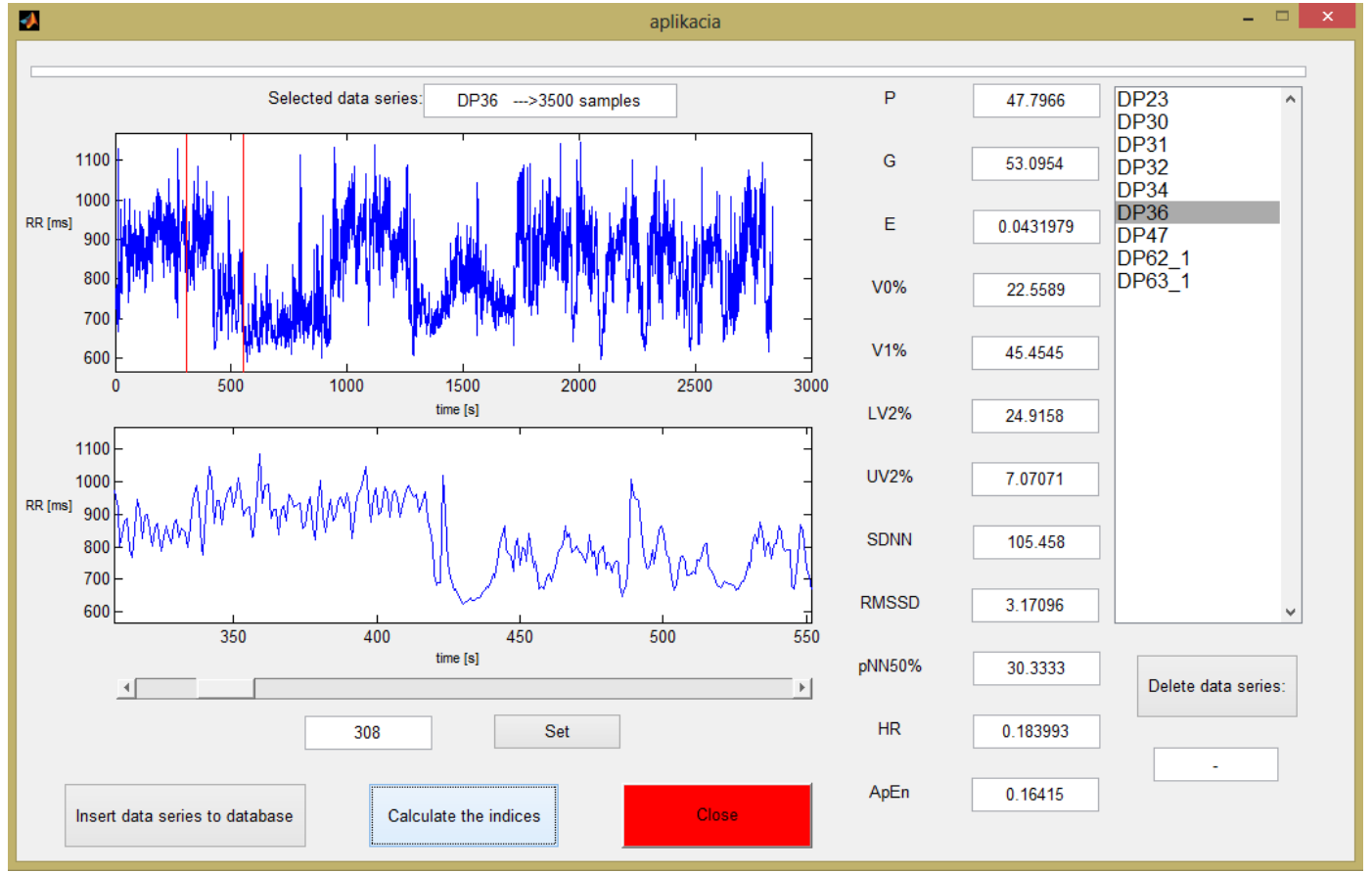

Fig. 5 GUI of heart rate variability tool for evaluation of autonomic nervous system function

\section{REFERENCES}

[1] CHIDA, Y. - STEPTOE, A.: Stress Reactivity and Its Association With Increased Cardiovascular Risk: A Role for the Sympathetic Nervous System?, Hypertension no. 55, 2010, doi:10.1161/HY PERTENSIONAHA.110.153841

[2] LOVALlO, W. R.: Do low levels of stress reactivity signal poor states of health? Biological Psychology, no. 86, 2011, pp. 121-128, doi: 10.1016/j.biopsycho.2010.01.006.

[3] GOLDBERGER, A. L. - PENG, C. K.. - LIPSITZ, L. A.: What is physiologic complexity and how does it change with aging and disease? , Neurobiology of Aging, no. 23, 2012, pp. 23-26, doi: 10.1016/S0197-4580(01)00266-4.

[4] BAR, K. J. et al.: Non-linear complexity measures of heart rate variability in acute schizophrenia, Clinical Neurophysiology, no. 118, 2007, pp. 20092015, doi: 10.1016/j.clinph.2007.06.012.

[5] BORNAS, X. et al.: Fear induced complexity loss in the electrocardiogram of flight phobics: a multiscale entropy analysis, Biological Psychology, no. 73, 2006, pp. 272-279, doi: 10.1016/j.biopsy cho.2006.05.004

[6] PORTA, A. et al.: An integrated approach based on uniform quantization for the evalution of complexity of short-term heart period variability: Application to $24 \mathrm{~h}$ Holter recordings in healthy and heart failure humnas, Chaos no. 17, 2007, doi: $10.1063 / 1.2404630$

[7] TARVAINEN, M. P. et al.: Kubios HRV - Herat rate variability analysis software, Computer Methods and Programes in Biomedicine, pp. 210-220, 2014
[8] RAMSHUR, J. T.: Design, evaluation and application of heart rate variability analysis software (HRVAS), A thesis Presented for the Master of Science Degree. Memphis: The University of Memphis, 2010, pp. 754-760.

[9] VOSS, A. et al.: The application of methods of nonlinear dynamics for the improved and predictive recognition of patients threatened by sudden cardiac death, Cardiovascular Research, no. 31, 1996, pp. 419-433, doi: 10.1016/S0008-6363(96)00008-9.

[10] HOU, F. - ZHUANG, J. - BIAN, Ch.: Analysis of heartbeat asymmetry based on multi-scale time irreversibility test, Physica A: Statistical Mechanics and its Applications, no. 389 , 2010, pp. 754-760, doi: 10.1016/j.physa.2009.10.003

[11] VOSS, A. et al.: Methods derived from nonlinear dynamics for analysing heart rate variability, Phylosophical Transaction of the Royal Society. 2009, no. 367, doi: 10.1098/rsta.2008.0232.

[12] PORTA, A.: Assessing complexity of short-term heart period variability through entropy-based approaches. Lecture, University of Milan, 2013.

[13] PORTA, A. et al.: Symbolic Analysis of Short-Term Heart Period Variabilty during Graded Head-Up Titl, Milan, Computers in Cardiology, 2006. ISSN 0276-6547.

[14] PORTA, A. et al.: Temporal asymmetries of shortterm heart period variability are linked to autonomic regulation, American Journal of Physiology, no. 295, 2008, doi: 10.1152/ajpregu.00129.2008.

[15] VISNOVCOVA, Z. - MESTANIK, M. JAVORKA, M. - MOKRA, D. - GALA, M. JURKO, A. - CALKOVSKA, A. TONHAJZEROVA, I.: Complexity and time 
asymmetry of heart rate variability are altered in acute mental stress, Physiological Measurement, 2014 May 22; 35(7): ISSN: 1319-1334, doi: 10.1088/0967-3334/35/7/1319.

Received May 31, 2014 , accepted August 13, 2014

\section{BIOGRAPHIES}

Gála Michal was born on 06.02.1983. In 2006 he graduated (MSc.) at the Department of Electromagnetic and Biomedical Engineering at University of Zilina in Zilina. In 2009 he graduated (Dr.) at the Department of Cybernetics and Biomedical Engineering at VSB Technical University of Ostrava. Since 2009 he is working at the Department of Electromagnetic and Biomedical Engineering at University of Zilina. His scientific research is focusing on biomedical image and signal processing, on artificial intelligence in biomedicine and on biological processes simulations.

Kadlecová Jana was born on 16.04.1990. In 2014 she graduated (MSc.) at the Department of Electromagnetic and Biomedical Engineering at University of Zilina in Zilina.

Višňovcová Zuzana was born on 30.6.1986. In 2010 she graduated (MSc) at the Department of Electromagnetic and Biomedical Engineering at University of Zilina in Zilina. Since 2010 she has studied a postgradual studium at the Department of Physiology at Jessenius Faculty of Medicine at Comenius University in Martin. The thesis title is "The effect of load on the autonomic nervous system". Her scientific research is focusing on monitoring changes in autonomic nervous system in response to mental load through the signals of heart rate, electrodermal activity and peripheral temperature using linear and nonlinear methods of analysis. 